\title{
Association between corneal arcus and some of the risk factors for coronary artery disease
}

\author{
JACOB PE'ER, ${ }^{1}$ JESUS VIDAURRI, ${ }^{1}$ SIMAN-TOV HALFON ${ }^{2}$ \\ SHLOMO EISENBERG, ${ }^{3}$ AND HANAN ZAUBERMAN ${ }^{1}$
}

From the ${ }^{1}$ Department of Ophthalmology, Hadassah University Hospital, the ${ }^{2}$ Department of Medical Ecology, Hebrew University-Hadassah Medical School, and the ${ }^{3}$ Lipid Research Laboratory, Department of Medicine B, Hadassah University Hospital, Jerusalem, Israel

SUMMARY The relationships between coronary artery disease risk factors and corneal arcus were examined in 150 adults aged 55 years and above of both sexes and from different ethnic origins. The width of the corneal arcus was measured accurately by a digitiser, and the risk factors for coronary artery disease were examined according to the standard procedure used by the Lipid Research Clinics. The results show that the corneal arcus is more frequent in males; the frequency and size of corneal arcus are positively associated with age; there is a positive correlation between the size of corneal arcus and the levels of cholesterol and low-density lipoprotein in males; and that there is negative correlation between corneal arcus and diastolic blood pressure in both sexes. No associations were found between corneal arcus and other coronary artery disease risk factors such as triglyceride, high-density lipoprotein, very low-density lipoprotein, weight, Quetelet's ratio, glucose, and smoking.

Corneal arcus is a common change of the aging cornea. Lipids accumulate at the periphery of the cornea (in the peripheral corneal stroma, Bowman's membrane, and Descemet's membrane) and form a ring-like opacity. A clear zone of about $1 \mathrm{~mm}$ remains between the corneal arcus and the limbus; the central part of the cornea is never involved. ${ }^{1}$

The corneal arcus is clinically harmless. Vision is not diminished, and ulcerations and vascularisation of the cornea do not occur. Corneal arcus is nevertheless interesting because of its occurrence with certain other diseases, such as hyperlipoproteinaemia, especially types II and III. ${ }^{2}$

Several investigators have reported that corneal arcus is more likely to occur in patients with coronary artery disease (CAD) than in healthy controls, especially those under 50 years of age, ${ }^{3-6}$ while another ${ }^{7}$ noted significant correlation between the degree of corneal arcus and the incidence of electrocardiographic abnormalities in elderly patients. Furthermore the clinical and pathological features of corneal arcus suggest that the lipid accumulation in

Correspondence to Dr. Jacob Pe'er, Department of Ophthalmology, Hadassah University Hospital, PO Box 12000, 91120 Jerusalem, Israel. the cornea (cholesterol and its esters, triglyceride, and phospholipids) depends on the blood vessels and that the primary alteration is localised in the pericorneal vessels. ${ }^{18}$ The fat content of a corneal arcus appears to be derived from the blood; other features as well are shared by arteriosclerosis and corneal arcus. ${ }^{5}$ It has therefore been popularly supposed that the occurrence of a corneal arcus might be associated with the presence of arteriosclerosis, though one author did postulate the opposite. 9 It is pertinent, then, to attempt to relate corneal arcus with other risk factors which may be associated with or predictive of coronary artery disease.

The availability of a group of patients previously studied for risk factors for ischaemic cardiovascular disease prompted us to screen them for the presence or absence of corneal arcus and to look for relationships between the corneal arcus and these risk factors.

\section{Patients and methods}

Subjects participating in the study were randomly selected from the Jerusalem Lipid Research Clinic (LRC) adult population. ${ }^{10}$ They underwent the LRC screen which included: detailed questionnaires; 
determinations of plasma total cholesterol and triglyceride (TG); determination of very low-density lipoprotein (VLDL), low-density lipoprotein (LDL), and high-density lipoprotein cholesterol levels (HDL); serum glucose and other biochemical variables; systolic and diastolic blood pressure (BP); anthropometric measurements: weight $(\mathrm{kg})$, height $(\mathrm{cm})$, the derived Quetelet's ratio (weight/ height ${ }^{2} \times 10000$ ), and triceps skin fold thickness $(\mathrm{mm})$; smoking history: nonsmokers and smokers (numbers of cigarettes per day). All these procedures were conducted in accordance with standard LRC protocols. ${ }^{10}$ " For analysis all of the above mentioned variables were treated as independent variables.

One hundred and fifty participants of both sexes were included in the present study, which was carried out during the years 1980-2. Their age was 55 years and above except for one male aged 46 years. The participants were invited on a voluntary basis, through letters of invitation and telephone reminders; almost all of those invited participated in the study.

The results of all the nonophthalmological examinations were hidden from the eye-examining team until the study was completed.

The examination of the eye was done with 2 purposes: to study the risk factors for drusen in the macular area (study submitted for publication), and to study the association of risk factors for coronary vascular disease and corneal arcus.

An ophthalmic history was taken from all subjects, and the following examinations were performed: (1) Visual acuity (VA) was measured with the patient's own optical correction. If the acuity was less than $20 / 20$ it was re-examined with a pinhole in front of each eye. A Snellen chart placed at 20 feet $(6 \mathrm{~m})$ and a Jaeger reading chart were used for distance and near vision respectively. (2) The anterior segment of the eye was checked with a Haag-Streit slit-lamp, and the intraocular pressure was measured with an applanation tonometer.

Pictures of the cornea were taken with a Zeiss photo slit-lamp camera, with magnification $\times 1$. The film used was Kodachrome, 25 Asa, $35 \mathrm{~mm}$. The slides of the cornea were projected onto a Sonic digitiser table and were magnified 30 times. The width of the corneal arcus was measured with the aid of a stylus of this digitiser that transferred the information into a PDP-15 computer which made the calculation.

The width of the arcus of the more affected eye at its widest point was taken into account in our statistical tests. The width of the corneal arcus is defined as the 'dependent variable.'

Statistical methods. Descriptive statistics including the mean, median, and range were given for each independent and dependent variable. Student's $t$ test was used to assess the differences between the independent variables-in 2 groups, with and without corneal arcus. The association between each of the independent variables and corneal arcus were calculated by Pearson's correlation. Stepwise multiple regression analysis was performed to evaluate the association of corneal arcus with some of the independent variables.

\section{Results}

In examining the distribution of the age, sex, and origin of the 105 males and 45 females under study we found the average age of males was 61.9 years and of females 59.0 years. Of the male population $64.8 \%$ were 61 years or older, while among the females only $28.9 \%$ were 61 or older. One-third of the males and $24.4 \%$ of the females presented with corneal arcus. The mean width of the corneal arcus was higher in the male group $(0.61 \mathrm{~mm}$ versus $0.53 \mathrm{~mm})$.

$50 \%$ of all eyes examined had $20 / 20$ visual acuity. Nine eyes had a VA of less than 20/200, and 6 eyes were blind. No significant differences in VA were found between the groups with and without corneal arcus in the $t$ test, and no correlation was found between VA and corneal arcus.

Two eyes (of 2 subjects) had intraocular pressures (IOP) of 24 and $27 \mathrm{mmHg}$ respectively. The other 292 eyes had IOP ranging from 8 to $22 \mathrm{mmHg}$. No significant differences in IOP were found between the groups with and without corneal arcus in the $t$ test, and no correlation was found between IOP and corneal arcus.

The variables (cholesterol, triglyceride, HDL, LDL, VLDL, weight, height, Quetelet's ratio, triceps skin fold, systolic and diastolic blood pressure, serum glucose, age, and number of cigarettes smoked per day) of subjects with and without corneal arcus were compared statistically by means of Student's $t$ test. A significant difference was found between the 2 groups in plasma cholesterol level and age. Interestingly the group with corneal arcus had lower mean systolic and diastolic blood pressures than the group without corneal arcus; this difference was statistically significant only for the diastolic blood pressure. The differences in the other variables were not significant.

The possible association between corneal arcus size and the coronary artery disease risk factors were examined by means of Pearson's correlation coefficient in males and females. A significant correlation was found in the male group for cholesterol $(p=0.004)$ and LDL $(p=0.015)$, indicating that probably part of this association is related to the cholesterol portion of the LDL. A strong negative correlation was observed for diastolic blood pressure in both sexes. There was also a 
negative correlation with the systolic blood pressure; however, this correlation was not statistically significant. We found a positive correlation between corneal arcus and age in both sexes; however, only for men was this correlation significant. The correlations between the arcus senilis and the other risk factors were not significant.

We examined in stepwise multiple regression analysis the possible association between corneal arcus as a dependent variable and cholesterol, weight, systolic and diastolic blood pressure, and age as independent variables. Three of the variables cholesterol, diastolic blood pressure, and age-had a significant coefficient in the male group, but none of them was significant in the female group.

\section{Discussion}

In attempting to establish a possible relationship between corneal arcus and some of the risk factors for coronary artery disease we have studied a group of 150 subjects who are under the LRC follow-up; all of them (except one) are above the age of 55 years.

Our results confirm the reports of others ${ }^{56}$ that corneal arcus appears more frequently in males than in females (33.3\% against $24.4 \%$ ), and in addition we found that the mean corneal arcus in males is wider than that in females $(0.61 \mathrm{~mm}$ versus $0.53 \mathrm{~mm})$, though the difference is not significant. The percentage of subjects with corneal arcus of both sexes in our study was much lower than in other studies, especially those that were done in Scotland ${ }^{5}$ and Finland. ${ }^{12}$

Frequency and size of corneal arcus were positively associated with age, as was shown in other studies $^{4-61314}$ and match the popular name-arcus senilis.

Moreover the significant correlation of corneal arcus with cholesterol in males confirms the results of other authors ${ }^{4-615}$ and the known fact that it is a common feature of familial hypercholesterolaemia. ${ }^{16}$ The significant correlation of LDL in males is in agreement with results of Rifkind ${ }^{5}$ and with the impression of François ${ }^{1}$ that there seems to be a genetic relationship between familial hyper- $\beta$ lipoproteinaemia, artherosclerosis, and corneal arcus. The correlation of cholesterol and LDL in the women was not significant and lowered the significance of the results of the total group. This may be explained by the fact that women have a similar LDL cholesterol but a higher HDL cholesterol level than men of the same age. ${ }^{17}$

The correlation between corneal arcus and cholesterol in our study is not so prominent as was found in other studies, ${ }^{4-5} 15$ though it is significant. This can be explained by the age of the subjects in our group
(55 and above), in which age had a more significant role than cholesterol level. Moreover these studies showed the strong relationship between corneal arcus, cholesterol, and coronary artery disease in the younger patients.

An interesting result not found in previous studies is the significant negative correlation with diastolic blood pressure in both sexes. Furthermore, in the group of patients with high diastolic BP $(100 \mathrm{mmHg}$ and above) not a single one had corneal arcus. Some studies $^{6913}$ have mentioned that there was no positive correlation between corneal arcus and blood pressure, but none mentioned a negative correlation, a result that was found in all our statistical analyses. The borderline correlation found in the present study with number of cigarettes smoked per day $(0.054$ in males) comes close to the positive relation that Rosenman et al. found in their study. ${ }^{6}$

In conclusion, our results show that there is an association between corneal arcus and several of the risk factors for coronary heart disease which we examined. It is well known that males are more vulnerable to coronary heart disease than females, that coronary heart disease is more frequent in the elderly, and that high levels of serum cholesterol and LDL are among its most important risk factors. To these we can add the positive influence of smoking on coronary heart disease. All these facts are expressed in the results we found in relation to corneal arcus except for smoking, which in our study had only a borderline significance. Further study is needed to explain the negative correlation of corneal arcus and diastolic blood pressure.

The authors acknowledge their thanks to Judith Fisher, Department of Ophthalmology, and the staff of the Department of Medical Illustration, Hadassah University Hospital, for their important contribution to this study.

\section{References}

1 François J. Arcus senilis. Doc Ophthalmol 1973; 34: 165-82.

2 Vinger PF, Sachs BA. Ocular manifestations of hyperlipoproteinemia. Am J Ophthalmol 1970; 70: 563-73.

3 Pomerantz HZ. The relationship between coronary heart disease and the presence of certain physical characteristics. Can Med Assoc J 1962; 86: 57-60.

4 Shanoff HM, Little JA. Studies of male survivors of myocardial infarction due to 'essential' atherosclerosis. III. Corneal arcus: incidence and relation to serum lipids and lipoproteins. Can Med Assoc J 1964; 91: 835-9.

5 Rifkind BM. The incidence of arcus senilis in ischaemic heart disease - its relation to serum lipid levels. Lancet 1965; i: 312-3.

6 Rosenman RH, Brand RJ, Sholtz RI, Jenkins CS. Relation of corneal arcus to cardiovascular risk factors and the incidence of coronary disease. N Engl J Med 1974; 291: 1322-4.

7 Rodstein M, Zeman FD. Arcus senilis and arteriosclerosis in the aged. Am J Med Sci 1963; 245: 70-7.

8 Cogan DG, Kuwabara T. Arcus senilis, its pathology and histochemistry. Arch Ophthalmol 1959; 61: 553-60.

9 White CE. The relation of arcus senilis to arteriosclerosis and senility. N Engl J Med 1935; 212: 10-3. 
10 Halfon ST, Friedlander Y, Halperin G, et al. Plasma high-density lipoprotein cholesterol, total cholesterol and triglyceride levels in 17 year-old Jewish residents of Jerusalem. Circulation 1980; 62 (suppl IV): 93-8.

11 Lipid Research Clinics Program. Manual of laboratory conditions. Vol. 1, Lipid and lipoprotein analysis. NHLBI, NIH, Bethesda, Md, 1974 (DHEW publication no. (NIH) 75-628).

12 Forsius H. Arcus senilis corneae. Acta Ophthalmol (Kbh) 1954; 42 (suppl): 1-78.

13 Hickey N, Maurer B, Mulcahy R. Arcus senilis: its relation to certain attributes and risk factors in patients with coronary heart disease. Br Heart J 1970; 32: 449-52.

14 McAndrew GM, Ogston D. Arcus senilis in middle-aged men. $\mathrm{Br}$ Med J 1965; i: 425-7.

15 Varnek L, Schnohr P, Jensen G. Presenile corneal arcus in healthy persons-a possible cardiovascular risk indicator in younger adults. Acta Ophthalmol (Kbh) 1979; 57: 755-65.

16 Winder AF. Factors influencing the variable development of corneal arcus and xanthelasmata in familial hypercholesterolemia. Presented at the Genetics in Ophthalmology conference, Jerusalem, September 1981.

17 Herss G, Tamir I, Davis CE, et al. Lipoprotein-cholesterol distribution in selected North American populations: the Lipid Research Clinics Program Prevalence Study. Circulation 1980; 61: 302-15. 